\title{
Objective versus Subjective: Kudzu Terminology
}

\author{
Jules M. Rothstein, PhD, PT, FAPTA (Editor, Physical Therapy)
}

\section{INTRODUCTION TO EDITORIAL}

The words subjective and objective are frequently encountered in clinical discussions and journal articles. But what do they mean? I suspect at this point you may be thinking, "Good grief! Is he going to devote an entire editorial to the obvious?" The answer to this question is both yes and no. Yes, I am going to devote an editorial to these terms; and, no, what you are about to read is not obvious-at least, it was not obvious for me when I first read it in 1996. It was written by Jules Rothstein, the editor of Physical Therapy at that time and an individual who was passionate about our profession and had a profound impact on physical therapy internationally. So sit back and be enlightened by words from the Master's pen. After reading this article, you may find yourself using these words differently.

This editorial is reprinted from Rothstein JM. Objective versus subjective: kudzu terminology. Phys Ther. 1996; 79:814-6, with the permission of the American Physical Therapy Association.

Paul Stratford, Editor

\section{OBJECTIVE VERSUS SUBJECTIVE: KUDZU TERMINOLOGY}

Kudzu, the ubiquitous weed of the southern United States, may be an immigrant to America, but it is a hardy immigrant almost immune to eradication. Cut it back, and it grows again; cut it again, and it's back again. Even natural enemies are destroyed by kudzu's capacity to propagate. Kudzu is to weeds what Jason (of "Friday the 13th" fame) is to campers and other unsuspecting victims-impossible to kill. Unfortunately, kudzu and Jason have a kindred analog in physical therapy: Our willingness to misuse the terms objective and subjective. These terms live in a malevolent state, as invincible as Jason in his hockey mask. Consider this Note one more attempt to cut back the kudzu and send Jason to a watery demise.

Subjective and objective have become as value laden as any terms one hears in clinical practice or research. Subjective has come to represent things less meaningful, whereas objective has come to represent things important. We use these terms without realizing that although phenomena can be subjective, the measurements themselves can be highly objective. There also are subjective measurements of objective phenomena, as in the case of manual muscle testing grades above what we traditionally called Fair. Confused? Don't blame me; blame those who fail to differentiate what the adjectives subjective and objective modify-and try to follow along!

Phenomena exist, and sometimes they have a recognizable physical reality, a tangible manifestation. Mass clearly has a physical manifestation, for instance, as do forces. Some phenomena seem to exist only as a conceptual basis, but even these sometimes influence behaviors and observable events. Inventories of emotions, self-worth, or quality of life are based on formulations (constructs) that define phenomena. Is there really a fundamental difference between phenomena that have physical reality and those that can be discerned only by attention to theoretical constructs? Yes and no!

As any physics student can tell you, there are definitions (constructs) that define phenomena that manifest a physical reality. They don't simply exist, they exist and can be identified because there are definitions that are universally agreed on. Mass is definable by a construct (in this case, a formula), as are forces, temperatures, and all similar phenomena. In other words, anything we want to measure has to be definable for it to be measured, and all things measurable have an underlying construct that defines what they are.

Complex phenomena such as hate, the capacity to work, functional status, or patient satisfaction are not easily defined, but if a definition (construct) is supplied, they can be measured. We too often call measurements of patient satisfaction, self-reports of disability, and pain "subjective." But why? If we believe that they are phenomena having no physical reality discernible by anyone other than the person making the report, why do we apply the adjective to the nature (quality) of the measurement rather than applying it to what we are measuring?

My pain-over the use of these terms, for examplecan be felt only by me, but anyone with a suitable instrument can measure it. (Through my prose, you the reader are seeing a real-world manifestation of my pain in my whining.) If the instrument is good enough, everyone measuring my pain will get a similar estimate (measure) 
of my pain. The phenomenon may be unique to me (a subjective experience I am having), but the measurement, by virtue of measuring things in a reliable manner, would be objective. The experience is mine, but the measurement is there for all to see and consider in their decision making. The adjectives subjective and objective can relate to the quality of the measurement (we certainly desire all measurements to be as objective as they can be), or to what is being measured (it does not matter whether the phenomenon is objective or subjective, as long as we are measuring something we care about).

If a measurement can be replicated and have a universally shared reality, that measurement is objective even when it is used to describe a subjective phenomenon. Measurements that are reliable are objective measurements, independent of what they measure. In this way, we describe the quality of the measurement as being reliable (good) or not reliable (not so good, or even useless). Some real-world phenomena, such as forces, can be measured in a manner that is dependent on the measurer. When this occurs, the phenomena may be objective (potentially universally observable), but the measurement is not-it is subjective! Manual muscle testing grades above Fair are subjective not because they call for the judgment of the examiner-judgmentbased measurements can be objective-but because research indicates that these judgments have poor reliability. If there was good reliability between observers, these judgments, even though they are made by different examiners, would be objective measurements.

Force measurements with a dynamometer, on the other hand, are objective not because a machine is used but because they are reliable between examiners, and they are subjective if they are not reliable between examiners. This illustrates that when we use subjective and objective, we need to make certain whether we are discussing the quality of the measurement (degree of reliability) or the phenomena being measured. Measurements that are subjective in quality don't tell us much, because the variability between examiners indicates that there is a lot of error in the measurements. In my opinion, measurements of subjective phenomena that have objective quality are probably the most useful of clinical measurements because they often relate more to issues of value to patients and payers. That is, they relate more to disability status, whereas measurements of objective phenomena frequently are made at the impairment level.

Why should we care about this semantic confusion? When we fail to recognize the importance of observational measures, patient-derived measures, and similar measures by demeaning them with a pejorative use of the word subjective, we eliminate a great deal of useful and meaningful clinical measurements. If I were to argue that pain reports and activity reports were "too subjective," I might feel a mistaken need to examine whether seemingly "more objective" measurements could be used to illustrate the benefits of treatments. I might argue for the exclusive use of measures of force or range of motion.

Lost in this false dichotomy would be the fact that the measures are different and tell us different things. If the patient seeks relief of pain, I would want a reliable (objective) measurement of this subjective phenomenon, or better yet I might want an objective measurement of the patient's subjective view as to whether he or she improved. This would tell me something about the benefit of treatment. On the other hand, measurements of force and motion could, if I am not careful, be subjective, because I might use unreliable measurements. More important, however, is the fact that even if these measurements are objective (reliable) in quality, they do not deal with patient goals but with changes in impairment status. This can be very important information, but it is different from what I would learn from those so-called "subjective" measurements.

If we can cut back the kudzu and kill Jason and start thinking about measurements without misusing the terms subjective and objective, we would focus on the most relevant issues. Are the measurements reliable? That is, how much error do they have? What do the measurements tell us, and which are best in research and clinical practice? Documentation of practice and evidence for outcomes require high-quality measurements, and many of these can be of subjective phenomena. Using terms properly frees us to get on with the job at hand.

\section{CONCLUDING COMMENTS}

Sadly for us all, the world of physical therapy lost Jules Rothstein to cancer in August 2005. More than any other physical therapist in the world, Jules influenced the elevation of our profession to one of international scientific significance and stature through his outstanding contributions to and stewardship of Physical Therapy as Editor, Editor-in-Chief, and subsequently Editorin-Chief Emeritus (1989-2005). During that time, Jules contributed more than 150 provocative and educational editor's notes like the one reprinted here. They are all worth reading!

Because of his outstanding international contributions to physical therapy, Jules posthumously received the Mildred Elson Award for International Leadership in 2007 from the World Confederation for Physical Therapy, the highest honour bestowed by the WCPT for "sustained and continued leadership over a career." As the WCPT website states in describing Jules, "His outlook was truly international and multiculturalchampioning cultural diversity in US physical therapy education programmes, encouraging international 
submissions to Physical Therapy and giving presentations advocating research and scholarship around the globe. His Editor's Notes in the journal have been a great source of inspiration to the profession, and will continue to be so. He is widely regarded as one of the most influential physical therapists of his time, and one who has had an international and lasting impact on research in the profession." ${ }^{1}$

Susan R. Harris, Editor in Chief

\section{REFERENCE}

1. World Confederation for Physical Therapy [homepage on the Internet]. London: The Confederation; 2007 [updated 2007 Aug 28; cited 2007 Nov 28]. International Leadership-Mildred Elson Award. Dr. Jules Rothstein [about 1 screen]. Available from: http://www.wcpt.org/ about/awards/2007.php.

D0l:10.3138/physio.60.2.103 